\title{
Use of capecitabine in management of early colon cancer
}

This article was published in the following Dove Press journal:

Cancer Management and Research

18 August 2011

Number of times this article has been viewed

\section{$\mathrm{H}$ Hameed \\ J Cassidy}

Beatson West of Scotland Cancer Centre, Glasgow, Scotland, UK
Correspondence: J Cassidy Beatson West of Scotland Cancer Centre, 1054 Great Western Road, Glasgow, UK G 12 OYN

Email jimcassidy 1958@live.co.uk
Abstract: Capecitabine (Xeloda ${ }^{\circledR}$, Roche, Basel, Switzerland) is a pro-drug of 5-fluorouracil (5-FU), and it is converted to 5-FU in the cancer cell by enzymatic degradation. The role of capecitabine in colorectal cancer has evolved in the last 15 years. In early trials in the metastatic setting, capecitabine has shown superior response rates compared with those achieved with 5-FU (Mayo Clinic regimen) (26\% vs 17\%), with equivalent progression-free survival and overall survival. In the adjuvant setting, the Xeloda in Adjuvant Colon Cancer Therapy (X-ACT) trial demonstrated that capecitabine as a single agent led to improvement in relapse-free survival (hazard ratio: $0.86,95 \%$ confidence interval: $0.74-0.99, P=0.04$ ) and was associated with significantly fewer adverse events than 5-FU plus leucovorin (LV, folinic acid). On the basis of the X-ACT trial, capecitabine was approved by the United States Food and Drug Administration, the National Institute for Clinical Excellence, and the Scottish Medicines Consortium as monotherapy for the adjuvant treatment of stage III colon cancer. The next step was to incorporate capecitabine into combination therapy. The XELOXA trial studied the combination of capecitabine and oxaliplatin (XELOX) vs 5-FU/LV and demonstrated 5-year disease-free survival of $66 \%$ for XELOX, compared with $60 \%$ for 5 -FU/LV. The toxicity profile was also quite comparable in the two arms. So both the single agent use of capecitabine as well as in combination with oxaliplatin can be considered as part of the standard of care in management of early colon cancer in appropriately selected patient groups.

Keywords: 5-fluorouracil, 5-FU, leucovorin, folinic acid, LV, XELOX, oxaliplatin, FOLFOX

\section{Colon cancer}

Colon cancer is the second most common cancer in Europe and the third most common in UK. ${ }^{1-3}$ The incidence of colon cancer has not changed a lot in recent time, but age-standardized mortality has improved in the last 30 years. ${ }^{3}$ This improvement is more pronounced in early colon cancer, where management usually consists of surgery followed by adjuvant treatment. ${ }^{4}$ Adjuvant treatment is fluoropyrimidine based, and it has now become a standard of care in the management of colon cancer.

\section{Prognostic/predictive markers to define adjuvant treatment of colon cancer}

The most important prognostic factors at primary diagnoses are TNM (tumor, nodes, and metastases) staging of disease and the ability to perform complete resection of all macroscopic disease. After surgery, the next question always is the need for adjuvant treatment and also the choice of chemotherapeutic agent. The goal of postoperative 
(adjuvant) therapy is to eradicate occult micrometastases present at time of surgery thereby increasing the cure rate. Poor prognosis is associated with lymphovascular space invasion, perineural invasion, perforation/ulceration of tumor, signet ring/mucinous histology, and number of lymph nodes involved..$^{5-7}$ Evidence at the moment favors use of adjuvant treatment in stage III colon cancer (lymph node positive disease). However, with stage II colon cancer, adjuvant treatment is a question of debate, as data only show modest 3\% 5-year survival benefit with adjuvant chemotherapy compared with observation alone. ${ }^{8}$

Many molecular markers have been speculated to affect outcome, and examples are microsatellite instability (MSI-H), tumor-DNA ploidy, chromosomal deletions, and mutated p53. The influence of microsatellite instability to predict response to adjuvant 5-fluorouracil (5-FU)-based chemotherapy is controversial, and so far the evidence is retrospective and conflicting, with some studies showing no effect on overall survival but others showing a trend toward lower or improved survival. Ribic et al demonstrated survival advantage with adjuvant chemotherapy in microsatellite stable colon cancer and no benefit for patients with microsatellite instability positive tumor. ${ }^{28}$ In contrast to above, Kim et al found no relationship between MSI and chemotherapy in terms of overall survival. ${ }^{29}$ Similarly, allelic loss on chromosome 18q and p53 mutation are also considered as poor prognostic signs, but incorporation of these molecular markers into clinical evidence is difficult at present on grounds of lack of robust evidence.

\section{History of adjuvant treatment with evolution of 5-FU}

Colon cancer was considered to be refractory to any cytotoxic treatment until 5-FU was designed, synthesized, and patented by Charles Heidelberger in $1957 .{ }^{9}$ 5-FU is a pyrimidine antagonist, and it functions to inhibit DNA synthesis by blocking the formation of normal pyrimidine nucleotides via thymidylate synthase inhibition.

Fluorouracil has been the established treatment for locally advanced and metastatic colorectal cancer for nearly four decades. Meta-analysis in the early 1990s showed that single-agent 5-FU has a reasonable efficacy in patients with metastatic colon cancer, providing a tumor response rate of $10 \%-15 \%$. The addition and modulation of fluorouracil by leucovorin ( $\mathrm{LV}$, folinic acid) lead to doubling of the response rate. ${ }^{10}$ Moving from metastatic settings to adjuvant, the study by Moertel et $\mathrm{al}^{11}$ was the first breakthrough trial which showed survival advantage in patients with stage III colon cancer with fluorouracil plus levamisole. It demonstrated a reduction in the recurrence rate by $40 \%(P<0.0001)$ and the death rate by $33 \%$ $(P=0.0007)$. In the same trial, levamisole on its own reduced the recurrence rate by only $2 \%$ and the death rate by only $6 \% .{ }^{11}$ Also, a meta-analysis in 1988 showed similar results, with a small survival benefit for patients receiving adjuvant 5-FU after surgery compared with surgery alone (odds ratio of death: 0.83, 95\% confidence interval: $0.70-0.98) .{ }^{10}$

In the same era, efforts were made to increase the antineoplastic activity of fluorouracil by adding a cytostatic agent which can modulate its mechanism of action. LV was the first success story, and IMPACT (The International Multicenter Pooled Analysis of Colon Cancer Trials), a meta-analysis, showed that treatment with 5-FU/LV after surgery was associated with a $22 \%$ reduction in death rate compared with surgery alone in stage III colon cancer. ${ }^{10,12}$

As treatment of colon cancer evolved more, research questions were raised about the method of infusion (continuous infusion vs bolus infusion) of 5-FU along with dosage of LV. Pan-European Trials in Adjuvant Colon Cancer (PETACC-2 Trial) had examined the above question and failed to show any difference in outcome between continuous infusional regimens (Mayo regimen) vs Bolus infusion regimen. However the toxicity profile favored the infusional regimen with lesser incidence of grade III/IV diarrhea, neutropenia, and mucositis. ${ }^{13}$ Similarly, trials did not show any survival benefit of high dose LV compared with low dose LV in combination with 5-FU. ${ }^{8}$

At the same time, oxaliplatin and irinotecan were showing activity and potential roles in management of colon cancer. Oxaliplatin shows synergy to 5-FU by downregulation of thymidylate synthase, and its role was established in Multicenter International Study of Oxaliplatin/Fluorouracil/Leucovorin in the Adjuvant Treatment of Colon Cancer (MOSAIC), where 2246 patients with stage II (40\%) or stage III (60\%) colon cancer were randomized to receive 6 months of infusional fluorouracil and LV with or without oxaliplatin. ${ }^{14-16}$ After a median follow-up of 49 months, the 4-year disease-free survival was statistically superior in those patients who received oxaliplatin and 5-FU/LV, compared with those who received 5-FU/LV (72.9\% vs 68.3\%) (see Table 1 and Figure 1).

In contrast to the promising infusional 5-FU/oxaliplatin regimen, an irinotecan/bolus 5-FU combination never lived up to the expectations in adjuvant settings. The PETACC-3 Trial was a randomized Phase III trial which compared use of biweekly infusional fluorouracil/LV alone or in combination with irinotecan in the adjuvant treatment of stage III colon cancer. A total of 3278 patients (945 with stage II disease; 2333 with stage III disease) were randomized to receive fortnightly infusional 5-FU/LV alone or with the addition of 
Table I MOSAIC trial results

\begin{tabular}{|c|c|c|c|c|c|c|}
\hline \multirow{2}{*}{$\begin{array}{l}\text { Median follow-up, } \\
\text { months }\end{array}$} & \multicolumn{2}{|c|}{ Disease-free survival, \% } & \multirow{2}{*}{$\begin{array}{l}\text { Reduction in risk } \\
\text { of recurrence, \% }\end{array}$} & \multicolumn{3}{|c|}{ Overall survival, $\%^{\mathrm{a}}$} \\
\hline & $\begin{array}{l}\text { FOLFOX4 } \\
(n=I 123)\end{array}$ & $\begin{array}{l}\text { 5-FU/LV } \\
(n=I \mid 23)\end{array}$ & & $P$-value & $\begin{array}{l}\text { FOLFOX4 } \\
(n=1 \mid 23)\end{array}$ & $\begin{array}{l}5-F U / L V \\
(n=I \mid 23)\end{array}$ \\
\hline 37.9 & 78.2 & 72.9 & 23 & 0.002 & 84 & 81.9 \\
\hline 48.6 & 75.9 & 69.1 & 24 & 0.0008 & 79.2 & 76.6 \\
\hline 56.2 & 76.4 & 69.8 & 23 & $<0.001$ & 80.2 & 77 \\
\hline
\end{tabular}

Abbreviations: 5-FU, 5-fluorouracil; FOLFOX, combination of folinic acid (leucovorin), 5-fluorouracil, and oxaliplatin; LV, leucovorin; MOSAIC, multicenter international study of oxaliplatin/fluorouracil/leucovorin in the adjuvant treatment of colon cancer.

irinotecan. At a median follow-up of 32 months, the 3-year disease-free survival was $62.9 \%$ for the irinotecan combination and $59.9 \%$ for those receiving 5 -FU/LV, a difference which was not statistically significant (hazard ratio: 0.89 [95\% confidence intervals: $0.77-1.03], P=0.107) .{ }^{30}$

\section{Capecitabine: pro-drug of 5-FU}

An oral preparation of 5-FU would have obvious benefits in terms of convenience for patients. Unfortunately, 5-FU is not suitable for oral administration because of variable bioavailability and unpredictable degradation in the gastrointestinal tract. Inter- and intra-individual variation in dihydropyrimidine dehydrogenase activity (the main catabolic enzyme for 5-FU), particularly in gastric mucosa, was also a reason for unsuitability of oral 5-FU. ${ }^{17}$ Capecitabine (Xeloda ${ }^{\circledR}$, Roche, Basel, Switzerland) was designed as a pro-drug of fluorouracil, which will deliver 5-FU selectively to the tumor minimizing systemic toxicities. After gastrointestinal absorption, capecitabine is hydrolyzed in the liver by carboxyl esterase to produce 5 -deoxy-5-fluorocytidine, and this moiety is then deaminated on its pyrimidine ring to produce 5 -deoxy-5-fluorouridine

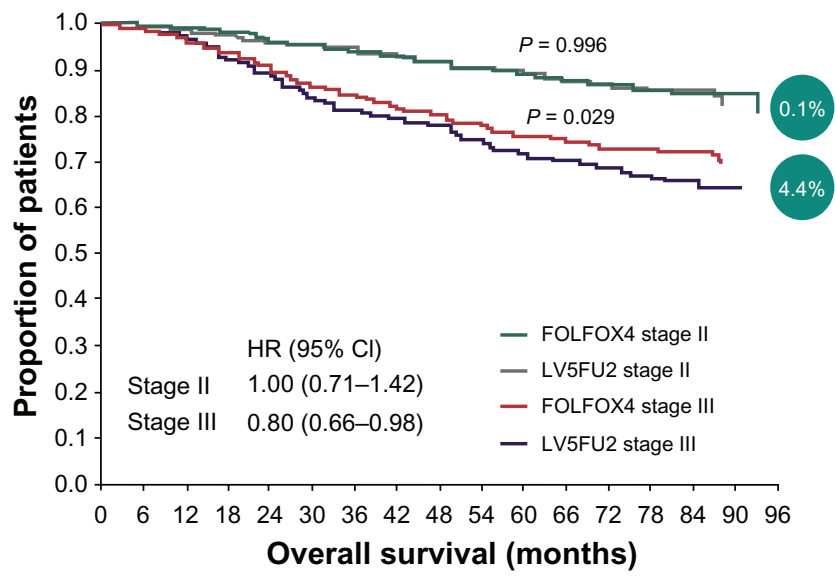

Figure I Overall survival in stage II vs stage III patients (4-year follow-up in MOSAIC trial).

Abbreviations: 5FU, 5-fluorouracil; $\mathrm{Cl}$, confidence interval; FOLFOX, combination of folinic acid (leucovorin), 5-fluorouracil, and oxaliplatin; HR, hazard ratio; LV, leucovorin; MOSAIC, multicenter international study of oxaliplatin/fluorouracil/ leucovorin in the adjuvant treatment of colon cancer. by cytidine deaminase, an enzyme located principally in hepatic and neoplastic tissue. The last enzymatic step of conversion of $5^{\prime}$-deoxy-5-fluorouridine to 5-FU in the cancer cell is catalyzed by thymidine phosphorylase, thus minimizing systemic exposure to 5-FU. Thymidine phosphorylase occurs at higher levels in most solid tumors than in the corresponding normal tissue. Human pharmacokinetic studies have demonstrated that capecitabine is well absorbed and rapidly converted to noncytotoxic intermediates and that intra-tumoral concentrations of 5-FU are significantly higher than plasma and normal tissue levels. ${ }^{18-20}$ Again, capecitabine completed the same cycle as was done with 5-FU, and it was tested in metastatic settings first where it showed good equivalent efficacy and better tolerability compared with 5-FU, and subsequently also in the adjuvant setting.

In early trials in the metastatic setting, capecitabine demonstrated response rate superior to those achieved with the Mayo Clinic regimen of 5-FU/LV (26\% vs 17\%), with equivalent progression-free survival and overall survival. ${ }^{21,22}$ Capecitabine was also better tolerated than 5-FU/LV, and its administration was associated with a reduced consumption of medical resources. ${ }^{27}$ As a result, capecitabine was considered as a good first-line treatment for metastatic colorectal cancer and became an established alternative to the combination of fluorouracil and LV.

The next challenge was to establish the role of capecitabine in adjuvant settings in colon cancer. The Xeloda in Adjuvant Chemotherapy Trial (X-ACT) investigated the efficacy and safety of capecitabine treatment vs 5-FU/LV treatment (Mayo Clinic regimen) in the postoperative adjuvant setting in 1987 patients with stage III (Dukes' C) colon cancer. This trial demonstrated that capecitabine was at least as effective as bolus 5-FU/LV in terms of disease-free and overall survival, with trends towards superiority for both. Moreover, there was less toxicity associated with capecitabine, apart from hand-foot syndrome, which was significantly more prevalent (Figures 2 and 3). On the basis of the X-ACT trial, capecitabine was approved by the United States Food and Drug Administration, the National Institute for Clinical Excellence, 


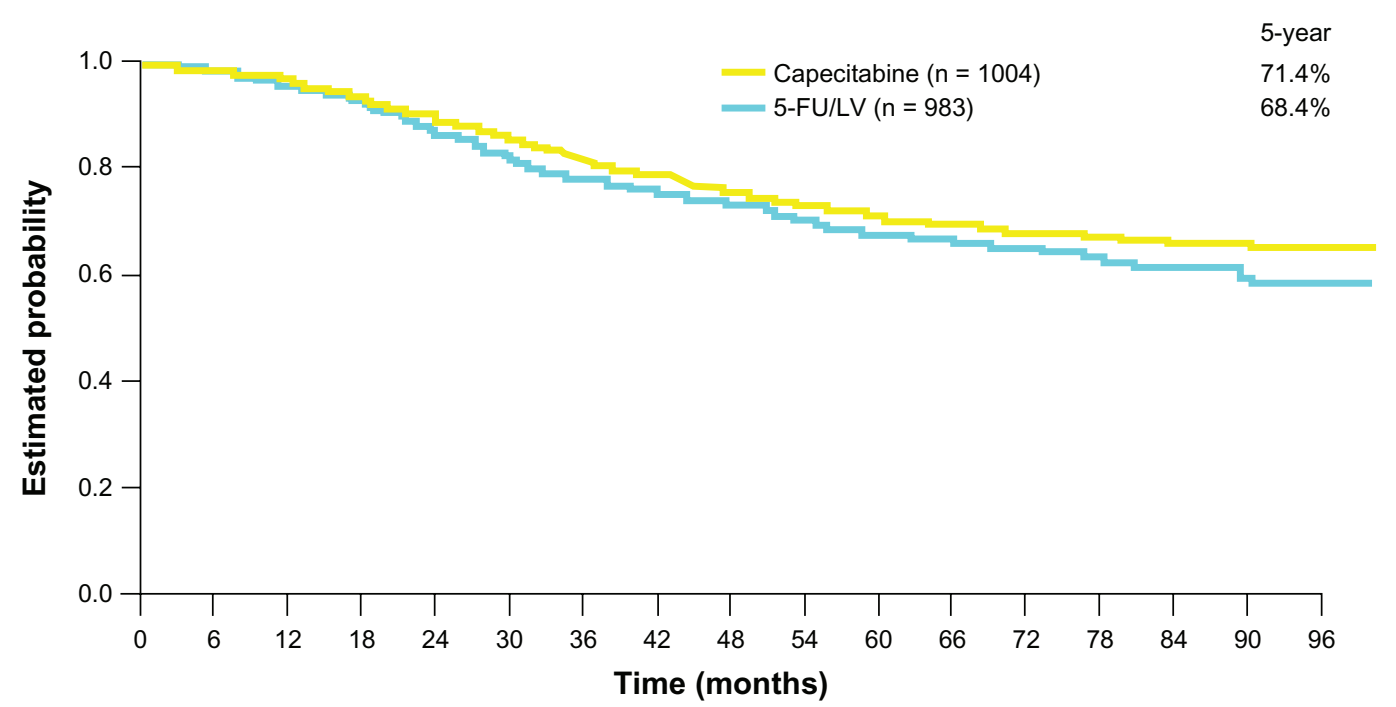

Test of noninferiority $P=0.000116$

Test of superiority $P=0.06$

\section{ITT population}

Figure 2 Overall survival curve (X-ACT trial).

Abbreviations: 5-FU, 5-fluorouracil; ITT, intention-to-treat; LV, leucovorin; X-ACT, xeloda in adjuvant colon cancer therapy.

and the Scottish Medicines Consortium as monotherapy for the adjuvant treatment of stage III colon cancer.

The next question was: does oxaliplatin also show synergy with capecitabine in a similar way as with 5-FU (MOSAIC trial). The XELOXA NO16968 trial answered the question. It recruited 1886 patients with stage III colon cancer at 226 study sites across 29 countries. They were randomly allocated to treatment with XELOX (oxaliplatin/capecitabine) or 5-FU/LV. This trial showed 5-year disease-free survival was $66 \%$ for XELOX vs $60 \%$ for $5-\mathrm{FU} / \mathrm{LV}$. The incidence of grade $3 / 4$ toxicities in the 5-FU/LV arm $(\mathrm{n}=924)$ and XELOX arm $(\mathrm{n}=937)$ were comparable (Figure 4$)^{23}$

Unfortunately, so far none of the "targeted" agents have shown efficacy in the adjuvant setting in colon cancer. A North Central Cancer Treatment Group randomized Phase III

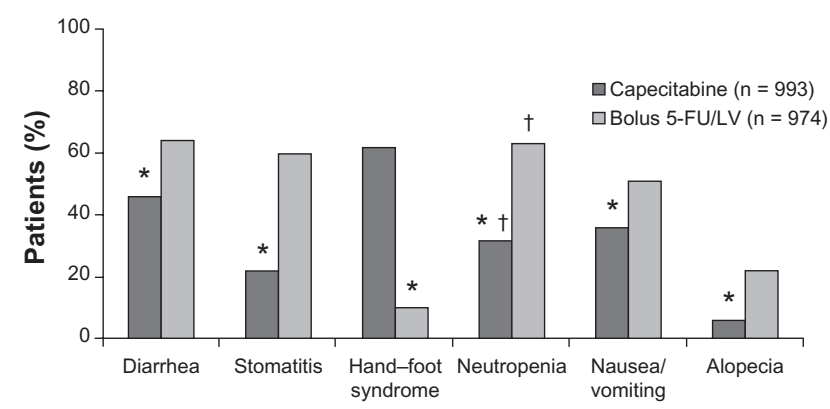

Figure 3 Safety profile of capecitabine vs 5-FU (all grades) (X-ACT trial). Notes: $* P<0.01$; †laboratary value.

Abbreviations: 5-FU, 5-fluorouracil; LV, leucovorin; X-ACT, xeloda in adjuvant colon cancer therapy. trial investigated the addition of cetuximab to mFOLFOX6 (modified LV, 5-FU, and oxaliplatin), and it showed no benefit for patients with resected stage III wild-type KRAS colon cancer. ${ }^{24}$ Recent trials with bevacizumab in combination with XELOX have failed to show any improvement in disease-free survival and overall survival in adjuvant therapy of colon cancer. $^{25,26}$

\section{Conclusion}

In conclusion, both the single agent use of capecitabine as well as in combination with oxaliplatin can be considered as part of the standard of care in management of early colon cancer in appropriately selected patient groups. Any role

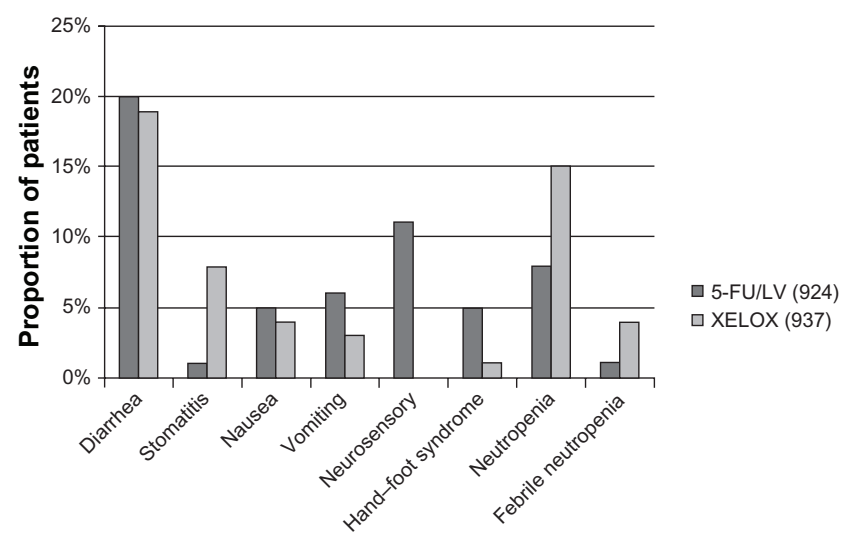

Figure 4 Incidence of grade III/IV toxicities in XELOXA trial.

Abbreviations: 5-FU, 5-fluorouracil; LV, leucovorin; XELOX, combination of capecitabine and oxaliplatin. 
of targeted treatments in combination with capecitabine is a research question, and such an approach cannot be recommended outside the context of clinical trials. We also have to wait for robust data regarding predictive/prognostic molecular markers in colon cancer and their role in defining need and choice of adjuvant treatment in colon cancer.

\section{Disclosure}

The authors report no conflicts of interest in this work.

\section{References}

1. Gray RF, Brewster DH, Kidd J, Burns H. Colorectal cancer in Scotland: recent trends in incidence and mortality. Gastrointest Oncol. 2002;4:213-222.

2. Hayne D, Brown R, McCormack M, et al. Current trends in colorectal cancer: site, incidence, mortality and survival in England and Wales. Clin Oncol. 2001;13:448-452.

3. Office for National Statistics. Cancer statistics - registrations, England, 2006. Series MB1 no. 37. London, UK: Office for National Statistics; 2008.

4. National Cancer Intelligence Network. Colorectal cancer survival by stage - NCIN Data Briefing. 2009 Jun. Available from: http://www. ncin.org.uk/publications/data_briefings/colorectal_cancer_survival_ by_stage.aspx. Accessed August 1, 2011.

5. Dukes CE. The classification of cancer of the rectum. J Pathol. 1932;35:323-332.

6. Beahrs OH, Henson DE, Hutter DE, et al. Manual for Staging of Cancer. 3rd ed. Philadelphia: Lippincott; 1988.

7. Edge SB, Byrd DR, Compton CC, et al. AJCC (American Joint Committee on Cancer) Cancer Staging Manual. 7th ed. Springer, New York; 2010:143.

8. QUASAR Collaborative Group. Comparison of fluorouracil with additional levamisole, higher dose folinic acid or both as adjuvant chemotherapy for colorectal cancer: a randomised trial. Lancet. 2000;355:1588-1596.

9. Heidelberger C, Chaudhuri NK, Danneberg P, et al. Fluorinated pyrimidines, a new class of tumour-inhibitory compounds. Nature. 1957; 179:663-666.

10. Modulation of 5-fluorouracil by leucovorin in patients with advanced colorectal cancer: evidence in terms of response rate. Advanced Colorectal Cancer Meta-Analysis Project. J Clin Oncol. 1992;10:896-903.

11. Moertel CG, Fleming TR, Macdonald JS, et al. Levamisole and fluorouracil for adjuvant therapy of resected colon carcinoma. $N$ Engl J Med. 1990;322:352-358.

12. International Multicentre Pooled Analysis of Colon Cancer Trials (IMPACT) Investigators. Efficacy of adjuvant fluorouracil and folinic acid in colon cancer. Lancet. 1995;345:939-944.

13. Carrato A, Kohne C, Bedenne L, et al. Folinic acid modulated bolus 5-FU or infusional 5-FU treatment of patients of UICC stage III colon cancer: preliminary analysis of the PETACC-2-study. Proc ASCO. 2006;24(18 Suppl):161s.

14. Goldberg R. Oxaliplatin in colorectal cancer: current studies. Oncology (Williston Park). 2000;14(12 Suppl 11):42-47.
15. Schmoll HJ, Cassidy J. Integrating oxaliplatin into the management of colorectal cancer. Oncologist. 2001;6 (Suppl 4):24-28.

16. André T, Boni C, Mounedji-Boudiaf L, et al; for the Multicenter International Study of Oxaliplatin/5-Fluorouracil/Leucovorin in the Adjuvant Treatment of Colon Cancer (MOSAIC) Investigators. Oxaliplatin, fluorouracil, and leucovorin as adjuvant treatment for colon cancer. N Engl J Med. 2004;350:2343-2351.

17. Fraile RJ, Baker LH, Buroker TR, et al. Pharmacokinetics of 5-fluorouracil administered orally, by rapid intravenous and by slow infusion. Cancer Res. 1980;40:2223-2228.

18. Milano G, Schellens JHM. Pyrimidine antimetabolites. In: Schellens JHM, McLeod HL, Newell DR, editors. Cancer Clinical Pharmacology. Oxford: University Press; 2005:51-62.

19. $\mathrm{Xu} \mathrm{Y,} \mathrm{Grem} \mathrm{JL.} \mathrm{Liquid} \mathrm{chromatography-mass} \mathrm{spectrometry} \mathrm{method}$ for the analysis of the anti-cancer agent capecitabine and its nucleoside metabolites in human plasma. J Chromatogr B Biomed Appl. 2003;783:273-285.

20. Twelves C, Glynne-Jones R, Cassidy J, et al. Effect of hepatic dysfunction due to liver metastases on the pharmacokinetics of capecitabine and its metabolites. Clin Cancer Res. 1998;4:941-948.

21. Cassidy J, Douillard JY, Twelves C, et al. Pharmacoeconomic analysis of adjuvant oral capecitabine vs intravenous 5-FU/LV in Dukes' C colon cancer: the X-ACT trial. Br J Cancer. 2006;94:1122-1129.

22. Van Cutsem E, Hoff P, Harper P, et al. Oral capecitabine versus intravenous 5-fluorouracil and leucovorin: integrated efficacy data and novel analyses from two large, randomised, phase III trials. Br J Cancer. 2004;90:1190-1197.

23. Schmoll HJ, Cartwright T, Tabernero J, et al. Phase III trial of capecitabine plus oxaliplatin as adjuvant therapy for stage III colon cancer: a planned safety analysis in 1,864 patients. J Clin Oncol. 2007;25:102-109.

24. Alberts SR, Sargent DJ, Smyrk TC, et al. Adjuvant mFOLFOX6 with or without cetuximab (Cmab) in KRAS wild-type (WT) patients (pts) with resected stage III colon cancer (CC): results from NCCTG Intergroup Phase III Trial N0147. J Clin Oncol. 2010:28(Suppl abstr CRA3507):262s.

25. Roche. Roche provides results on Avastin in adjuvant colon cancer [Press release 2010 Sep 18]. Roche, Basel, Switzerland.

26. Allegra CJ, Yothers G, O’Connell MJ, et al. Phase III trial assessing bevacizumab in stages II and III carcinoma of the colon: results of NSABP protocol C-08. J Clin Oncol. 2011:29:11-16.

27. Twelves C, Boyer M, Findlay M, et al. Capecitabine (Xeloda ${ }^{\mathrm{TM}}$ ) improves medical resource use compared with 5-fluorouracil plus leucovorin in a phase III trial conducted in patients with advanced colorectal carcinoma. Eur J Cancer. 2001:37:597-604.

28. Ribic CM, Sargent DJ, Moore MJ, et al. Tumor microsatellite-instability status as a predictor of benefit from fluorouracil-based adjuvant chemotherapy for colon cancer. N Engl J Med. 2003;349:247-257.

29. Kim GP, Colangelo LH, Wieand HS, et al. Prognostic and predictive roles of high-degree microsatellite instability in colon cancer: a National Cancer Institute-National Surgical Adjuvant Breast and Bowel Project collaborative study. J Clin Oncol. 2007;25:767-772.

30. Van Cutsem E, Labianca R, Bodoky G, et al. Randomized phase III trial comparing biweekly infusional fluorouracil/leucovorin alone or with irinotecan in the adjuvant treatment of stage III colon cancer: PETACC-3. J Clin Oncol. 27:3117-3125.
Cancer Management and Research

\section{Publish your work in this journal}

Cancer Management and Research is an international, peer-reviewed open access journal focusing on cancer research and the optimal use of preventative and integrated treatment interventions to achieve improved outcomes, enhanced survival and quality of life for the cancer patient The journal welcomes original research, clinical \& epidemiological

\section{Dovepress}

studies, reviews \& evaluations, guidelines, expert opinion \& commentary, case reports \& extended reports. The manuscript management system is completely online and includes a very quick and fair peerreview system, which is all easy to use. Visit http://www.dovepress.com/ testimonials.php to read real quotes from published authors. 